Research Paper

\title{
Erectile dysfunction and the risk of prostate cancer
}

\author{
Wei-Yu Lin 1,2,3, Ying-Hsu Chang ${ }^{4}$, Cheng-Li Lin ${ }^{5,6}$, Chia-Hung Kao ${ }^{7,8,9}$ and Hsi-Chin Wu ${ }^{10,11}$ \\ ${ }^{1}$ Division of Urology, Department of Surgery, Chang Gung Memorial Hospital, Gia-Yi, Taiwan \\ ${ }^{2}$ Chang Gung University of Science and Technology, Chia-Yi, Taiwan \\ ${ }^{3}$ Department of Medicine, Chang Gung University, Taoyuan, Taiwan \\ ${ }^{4}$ Division of Urology, Department of Surgery, Chang Gung Memorial Hospital, LinKo, Taiwan \\ ${ }^{5}$ Management Office for Health Data, China Medical University Hospital, Taichung, Taiwan \\ ${ }^{6}$ School of Medicine, China Medical University, Taichung, Taiwan \\ ${ }^{7}$ Graduate Institute of Clinical Medical Science, College of Medicine, China Medical University, Taichung, Taiwan \\ ${ }^{8}$ Department of Nuclear Medicine and PET Center, China Medical University Hospital, Taichung, Taiwan \\ ${ }^{9}$ Department of Bioinformatics and Medical Engineering, Asia University, Taichung, Taiwan \\ ${ }^{10}$ Department of Urology, China Medical University Hospital, Taichung, Taiwan \\ ${ }^{11}$ Graduate Institute of Clinical Medical Science and School of Medicine, College of Medicine, China Medical University, \\ Taichung, Taiwan \\ Correspondence to: Hsi-Chin Wu, email: wuhc@mail.cmuh.org.tw \\ Keywords: Prostate cancer (PCa), erectile dysfunction (ED), Cohort study, National Health Insurance Research Database, \\ malignancy \\ Received: October 19, $2016 \quad$ Accepted: March 21, $2017 \quad$ Published: April 13, 2017 \\ Copyright: Lin et al. This is an open-access article distributed under the terms of the Creative Commons Attribution License 3.0 \\ (CC BY 3.0), which permits unrestricted use, distribution, and reproduction in any medium, provided the original author and source \\ are credited.
}

\section{ABSTRACT}

Background: Prostate cancer (PCa) is the most commonly diagnosed malignancy and the third leading cause of cancer death among men in developed countries. Because some risk factors are common between erectile dysfunction (ED) and PCa, we investigated the association between ED and subsequent PCa.

Methods: This nationwide population-based cohort study used data from the Taiwan National Health Insurance Research Database for the period 2000-2010. We identified patients newly diagnosed with ED by using codes from the International Classification of Diseases, Ninth Revision, Clinical Modification.

Results: In total, 5858 and 23432 patients were enrolled in the ED and non-ED cohorts, respectively. After adjustment for age, sex, and comorbidities, the overall incidence densities of PCa were significantly higher in the ED cohort than in the nonED cohort, with an adjusted hazard ratio (aHR) of 1.19. The age-specific relative risk of PCa was significantly higher for all age groups in the ED cohort than in the non-ED cohort. Compared with patients without ED, those with organic ED had a 1.27-fold higher risk of PCa.

Conclusion: ED is a harbinger of PCa in some men. Physicians should consider the possibility of occult PCa in patients with ED regardless of age and comorbidities.

\section{INTRODUCTION}

Prostate cancer $(\mathrm{PCa})$ is the most commonly diagnosed malignancy and the third leading cause of cancer death among men in developed countries [1]. In the United
States alone, an estimated 242000 men were diagnosed with $\mathrm{PCa}$ in 2012, and 28,000 of them will die from the disease $[1,2]$. In Asia, although a trend of stage migration to the earlier stage has been observed in some countries, a relatively high rate of advanced $\mathrm{PCa}$ exists compared with 
the rates in the United States and Europe. This difference is mainly attributed to the lack of mass screenings [3-6].

Despite the high incidence and mortality of $\mathrm{PCa}$, few risk factors have been identified for the disease. Among those that have are older age, smoking, and obesity [7].Further investigation of the risk factors and their associated mechanisms of carcinogenesis is crucial to develop strategies for preventing and treating PCa [8].

Sexual activity is hypothesized as affecting prostate carcinogenesis, though the association between ejaculatory frequency and subsequent risk of $\mathrm{PCa}$ is controversial $[9,10]$. A previous study revealed that compared with men with erectile dysfunction (ED) who were not treated with phosphodiesterase type 5 inhibitors (PDE5i), men with ED who were treated with PDE-5i tended to exhibit a lower likelihood of being diagnosed with $\mathrm{PCa}$ because they were expected to exhibit higher ejaculation frequency than those not treated with PDE5i [11].

The risk of ED is related to many factors including age, smoking, diabetes, heart disease, depression, and hypertension [12-14].

$\mathrm{PCa}$ and ED share some common risk factors, including age, smoking, and obesity. In addition, ED is correlated with advanced $\mathrm{PCa}$ [15]. Furthermore, a previous study suggested that preoperative ED is a surrogate for adverse PCa outcomes following radical prostatectomy [16]. We postulate that ED is a sentinel symptom in patients with occult PCa. Thus, to examine this hypothesis, we performed a large-scale nationwide controlled cohort study in Taiwan to investigate whether $\mathrm{ED}$ increases the risk of $\mathrm{PCa}$ [17].

\section{RESULTS}

We included 5857 patients in the ED cohort and 5857 patients in the non-ED cohort. The age, occupation, urbanization level, and comorbidities distributions of the cohorts were similar (Table 1). The largest age subgroup of the men in both cohorts was $\leq 49$ years old $(39.3 \%$ vs. $39.9 \%$ ), and most of the men were white-collar workers $(55.0 \%$ vs $56.3 \%)$ and lived in an urbanized area $(66.5 \%$ vs $67.6 \%$ ). The mean frequency of medical visits/per year was $22.1(\mathrm{SD}=18.1)$ and $21.5(\mathrm{SD}=20.6)$ years for the $\mathrm{ED}$ and non- ED cohorts, respectively. The mean age of the ED and non-ED cohorts was 53.6 and 53.1 years, respectively. The cumulative incidence curves of PCa revealed that the ED cohort had a significantly higher risk of $\mathrm{PCa}$ than did the non-ED cohort $(P<.001$, log-rank test) (Figure 1).

The overall incidence densities of $\mathrm{PCa}$ were significantly higher in the ED cohort than in the nonED cohort (2.02 vs 1.63 per 1000 person-y), with an adjusted HR (aHR) of $1.19(95 \% \mathrm{CI}=1.08-1.31)$ after controlling for age; frequency of medical visits/per year; occupation category; urbanization level; comorbidities of hyperlipidemia, diabetes, hypertension, urinary stones, urinary tract infection, obesity, anxiety, and depression; and PCa screening strategies of prostate biopsy and TURP (Table 2). The age-specific relative risk of PCa was higher for age $<64$ y groups $(\mathrm{aHR}=1.49,95 \% \mathrm{CI}=1.32-1.69)$ in the ED cohort than in the non-ED cohort. The occupation category-specific analyses showed that the patients in the ED cohort had a significantly higher risk of PCa than did those in the non-ED cohort for white-collar workers. Patients in the ED cohort had a significantly higher risk of PCa than did those in the non-ED cohort for $2^{\text {th }}$ or $3^{\text {th }}$ urbanization level. The relative risk of PCa for the ED cohort compared with the non-ED cohort was significantly higher for both men without comorbidity (aHR $=1.95$, $95 \% \mathrm{CI}=1.65-2.30)$. In both cohorts, undergoing prostate biopsy or TURP was associated with an increased incidence of PCa. Among the patients without prostate biopsy, men with ED had a higher risk of PCa than did those without $\mathrm{ED}(\mathrm{aHR}=1.43,95 \% \mathrm{CI}=1.28-1.60)$. Similar results were observed for the patients without TURP; the ED cohort had a 1.53-fold higher risk of PCa than did the non-ED cohort $(95 \% \mathrm{CI}=1.38-1.70)$. Compared with the patients without $\mathrm{ED}$, those with organic ED had a 1.27-fold higher risk of PCa (95\% CI $=1.15-1.40)$ (Table 3).

The aHR of PCa slightly decreased as the follow-up duration increased (Table 4). Throughout the follow-up period, the ED cohort exhibited a higher risk of $\mathrm{PCa}$ than did the non-ED cohort, except for follow-up period $>5$.

\section{DISCUSSION}

Our data suggest that after adjustment for potential confounding factors, the risk of $\mathrm{PCa}$ was significantly higher in men with ED than in those without ED. PCa is the third leading cause of cancer death among men in developed countries $[2,18]$.In Asia, because of the lack of mass screenings, a high rate of advanced $\mathrm{PCa}$ is detected compared with the rate in the United States and Europe [4-6]. Hence, the identification of a predictive symptom or finding would enable even earlier intervention, possibly further reducing morbidity and mortality caused by the disease $[14,19]$. Such a predictor would be particularly useful for men who do not receive regular $\mathrm{PCa}$ assessments.

This study revealed that compared with men without ED, those with ED exhibited a higher risk of subsequent $\mathrm{PCa}$, regardless of age, comorbidities, and urbanization level. The risk of PCa was also higher in white-collar workers. The risk of $\mathrm{PCa}$ in our study population is significantly higher than that in the general population, even for a follow-up duration of more than 5 years. This finding may be partially attributable to the higher rate of prostate-related surgery (prostate biopsy plus TURP) in patients with ED (Table 1), which may result from more frequent contact with health care providers, leading to increased PCa screening and detection.

In order to decrease the surveillance bias, which resulted from more frequent contact with health providers because of other co-morbidities, We re-created a nonED cohort well matched for age, frequency of medical visits/per year, occupation, urbanization level, index year 
Table 1: Comparison of demographic characteristics and comorbidity of patients with ED and controls

\begin{tabular}{|c|c|c|c|c|c|}
\hline & \multicolumn{2}{|c|}{ Erectile dysfunction $(\mathrm{N}=\mathbf{5 8 5 7})$} & \multicolumn{2}{|c|}{ Control $(\mathrm{N}=5857)$} & \multirow[t]{2}{*}{ p-value } \\
\hline & $\mathbf{n}$ & $\%$ & $\mathbf{n}$ & $\%$ & \\
\hline Age, year & & & & & 0.04 \\
\hline$\leq 49$ & 2300 & 39.3 & 2337 & 39.9 & \\
\hline $50-64$ & 2273 & 38.8 & 2149 & 36.7 & \\
\hline$\geq 65$ & 1284 & 21.9 & 1371 & 23.4 & \\
\hline Mean $(\mathrm{SD})^{\#}$ & 53.6 & 13.6 & 53.1 & 14.4 & 0.06 \\
\hline $\begin{array}{l}\text { Frequency of medical visits/ } \\
\text { per year }\end{array}$ & 22.1 & 18.1 & 21.5 & 20.6 & 0.001 \\
\hline Occupation & & & & & 0.23 \\
\hline White collar & 3219 & 55.0 & 3289 & 56.3 & \\
\hline Blue collar & 1617 & 27.6 & 1539 & 26.3 & \\
\hline Others & 1021 & 17.4 & 1019 & 17.4 & \\
\hline Urbanization level $^{\dagger}$ & & & & & 0.05 \\
\hline 1 (highest) & 2038 & 34.8 & 2098 & 35.8 & \\
\hline 2 & 1854 & 31.7 & 1871 & 31.9 & \\
\hline 3 & 1014 & 17.3 & 1043 & 17.8 & \\
\hline 4(lowest) & 951 & 16.2 & 845 & 14.4 & \\
\hline \multicolumn{6}{|l|}{ Comorbidity } \\
\hline Hyperlipidemia & 1814 & 31.0 & 1837 & 31.4 & 0.65 \\
\hline Diabetes & 923 & 15.8 & 968 & 16.5 & 0.26 \\
\hline Hypertension & 2375 & 40.6 & 2386 & 40.7 & 0.84 \\
\hline Urinary stones & 508 & 8.67 & 533 & 9.10 & 0.42 \\
\hline Urinary tract infection & 303 & 5.17 & 274 & 4.68 & 0.22 \\
\hline Coronary artery disease & 1341 & 22.9 & 1310 & 22.4 & 0.49 \\
\hline Depression & 470 & 8.02 & 396 & 6.76 & 0.01 \\
\hline \multicolumn{6}{|l|}{ Prostate cancer screen } \\
\hline Prostate biopsy & 107 & 1.83 & 97 & 1.66 & 0.48 \\
\hline TURP & 155 & 2.65 & 146 & 2.49 & 0.60 \\
\hline
\end{tabular}

Chi-square test compared to total SD; ${ }^{\#} t$ test

NTD, New Taiwan dollar.

$\uparrow$ : The urbanization level was categorized into 4 levels according to the population density of the residential area, with level 1 indicating the highest urbanization and level 4 indicating the least urbanization.

*: Other occupation categories included primarily retired, unemployed, and low-income populations.

and comorbidities including hyperlipidemia, diabetes, hypertension, urinary stones, urinary tract infection, coronary artery disease, depression, prostate cancer screen, prostate biopsy, and TURP (Table 1). With this newly selected non-ED cohort, the associations between ED and PCa remain significant, although the HR decreases from 1.41 to 1.19 (Table 2).

The relationship between ED and subsequent diagnosis of PCa has not been investigated. Most studies have investigated the prevalence of ED before curative treatments for $\mathrm{PCa}$ through retrospective analysis $[15,16,20]$. To the best of our knowledge, this is the first large-scale nationwide cohort study to assess the relationship between ED and the risk of subsequent PCa.

In the present study, 5279 of the 5858 patients with ED (90.1\%) had organic ED (Table 3), which includes neurologic, hormonal, arterial, and cavernosal impairment 
etiologies. In addition, the cultural taboo in Asia against discussing sexuality should be considered [17]. A previous study suggested that ED is correlated with advanced $\mathrm{PCa}$ [15]. In the current study, the aHR for PCa in men with organic ED was $1.27(95 \% \mathrm{CI}=1.15-1.40)$ compared with that of men with psychogenic ED $(0.43,95 \% \mathrm{CI}=$ 0.13-1.37). Notably, among the men who had ED before PCa diagnosis, organic ED was the most common type. This result is different to the finding that a mix of organic and psychogenic ED is a common complication following prostate cancer treatment among men with PCa [15].

Our finding of a positive association between ED and subsequent diagnosis of $\mathrm{PCa}$ in follow-up duration of less than 1 year does not support an etiological role for ED in initiating prostate carcinogenesis because $\mathrm{PCa}$ tends to be slow growing with a protracted latency period; up to 20-30 years are required before the disease appears clinically $[21,22]$. Thus, it is plausible to assume the coexistence of ED and occult $\mathrm{PCa}$ rather than initiation of new-onset PCa. Therefore, our results may provide indications to aid clinical physicians in deciding whether to assess the risk of PCa in patients with ED.

On the other side, a high prevalence of ED has been observed in patients with chronic prostatitis/chronic pelvic pain syndrome (CP/CPPS), and a systemic review and meta-analysis confirmed a close association between ED and CP/CPPS $[17,23]$. In addition, meta-analyses of case-control studies have reported statistically significant associations between $\mathrm{PCa}$ and prostatitis (odds ratio = 1.6) $[8,24,25]$. Therefore, CP/CPPS may play a critical role in contributing to $\mathrm{ED}$ and subsequent $\mathrm{PCa}$ because pathological studies have shown that inflammation may be involved in the development of PCa $[7,26]$.

Moreover, compared with men without ED, the aHR for PCa $(1.19,95 \% \mathrm{CI}=1.08-1.31)$ was still significantly higher in men with ED, even for a follow-up duration of more than 5 years. We assume that patients with ED might lose the protective effects of frequent ejaculation on the risk of PCa. Such effects include modulated prostate carcinogenesis through the altering of the composition of prostatic fluid; decreased intraprostatic concentration of xenobiotic compounds and chemical carcinogens, which readily accumulate in prostatic fluid [27, 28]; and the reduced development of intraluminal prostatic crystalloids $[8,29]$.

This study has some limitations. First, no data on the International Index of Erectile Function Questionnaire or penile blood vessel examinations were available regarding the diagnosis of ED in this administrative database. Therefore, we could not analyze the association between the

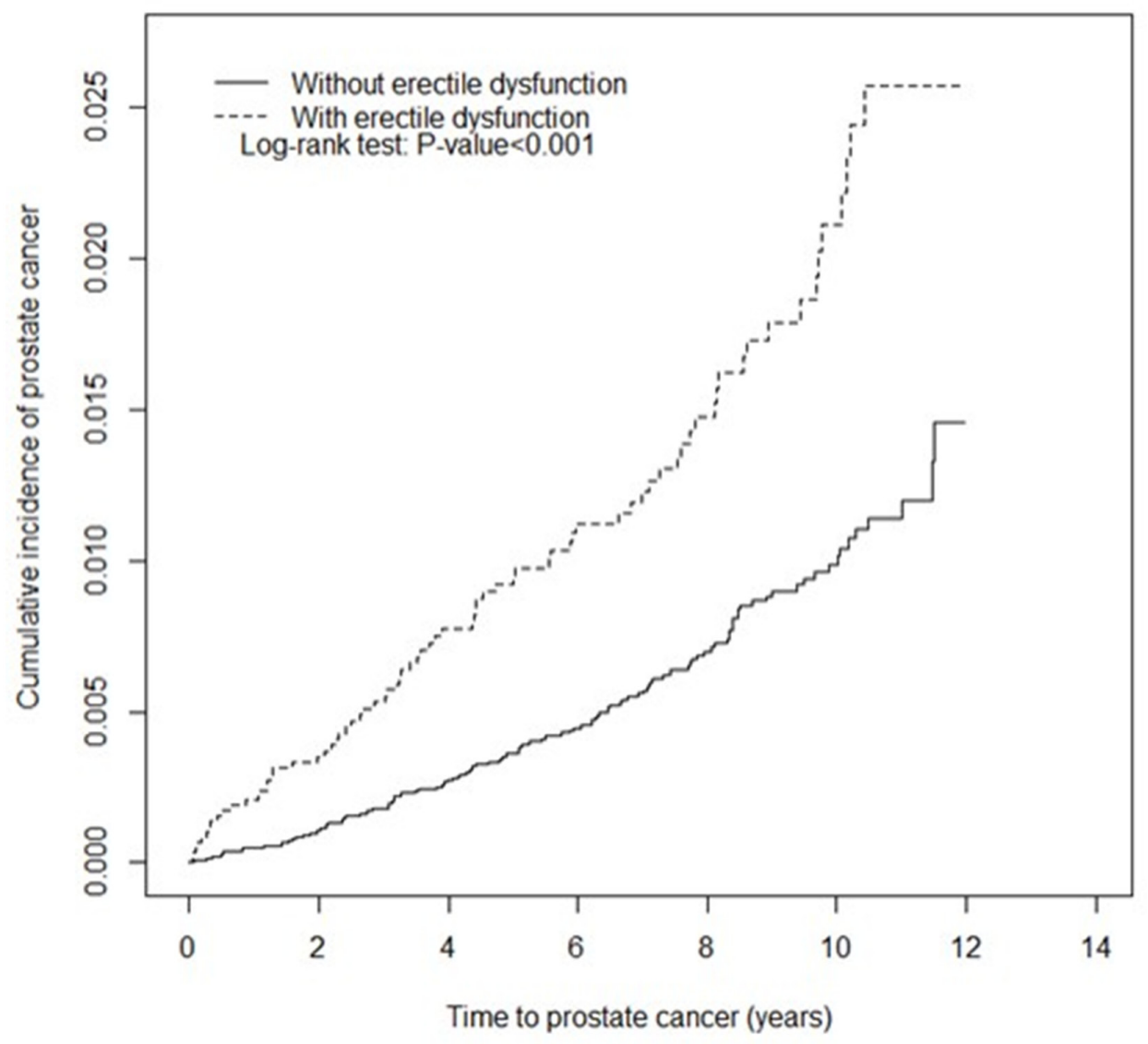

Figure 1: Kaplan-Meier curve of the cumulative incidence of prostate cancer in the cohorts of patients with and without erectile dysfunction. 
Table 2: Comparison of the incidence densities and hazard ratios of prostate cancer of men with and without erectile dysfunction stratified by demographic characteristics, comorbidity, prostate cancer screening, and medication

\begin{tabular}{|c|c|c|c|c|c|c|c|c|}
\hline & \multicolumn{6}{|c|}{ Erectile dysfunction } & \multirow[b]{3}{*}{ Crude HR (95\% CI) } & \multirow[b]{3}{*}{$\begin{array}{c}\text { Adjusted HR } \mathbf{H R}^{\dagger}(95 \% \\
\text { CI) }\end{array}$} \\
\hline & \multicolumn{3}{|c|}{ Yes } & \multicolumn{3}{|c|}{ No } & & \\
\hline & Event & PY & Rate $^{\#}$ & Event & PY & Rate $^{\#}$ & & \\
\hline All & 76 & 37603 & 2.02 & 59 & 36221 & 1.63 & $1.24(1.10,1.39)^{* * *}$ & $1.19(1.08,1.31)^{* * *}$ \\
\hline \multicolumn{9}{|l|}{ Age } \\
\hline$\leq 64$ & 28 & 29092 & 0.96 & 17 & 28218 & 0.60 & $1.60(1.38,1.84)^{* * *}$ & $1.49(1.32,1.69)^{* * *}$ \\
\hline$\geqq 65$ & 48 & 8511 & 5.64 & 42 & 8004 & 5.25 & $1.08(0.86,1.34)$ & $0.99(0.81,1.20)$ \\
\hline \multicolumn{9}{|l|}{ Occupation } \\
\hline White collar & 36 & 19998 & 1.80 & 25 & 20534 & 1.22 & $1.48(1.26,1.74)^{* * *}$ & $1.41(1.23,1.61)^{* * *}$ \\
\hline Blue collar & 15 & 10428 & 1.44 & 19 & 9508 & 2.00 & $0.71(0.36,1.39)$ & $0.97(0.49,1.94)$ \\
\hline Others & 25 & 7178 & 3.48 & 15 & 6179 & 2.43 & $1.44(1.10,1.88)^{* *}$ & $1.05(0.83,1.33)$ \\
\hline \multicolumn{9}{|c|}{ Urbanization level } \\
\hline 1 (highest) & 27 & 13139 & 2.06 & 24 & 12926 & 1.86 & $1.11(0.91,1.35)$ & $0.92(0.79,1.08)$ \\
\hline 2 & 26 & 11805 & 2.20 & 14 & 11796 & 1.19 & $1.86(1.49,2.31)^{* * *}$ & $1.35(1.12,1.63)^{* *}$ \\
\hline 3 & 12 & 6546 & 1.83 & 9 & 6325 & 1.42 & $1.29(0.98,1.69)$ & $1.68(1.33,2.12)^{* * *}$ \\
\hline 4 (lowest) & 11 & 6113 & 1.80 & 12 & 5175 & 2.32 & $0.78(0.58,1.04)$ & $1.00(0.78,1.27)$ \\
\hline \multicolumn{9}{|l|}{ Comorbidity ${ }^{*}$} \\
\hline No & 12 & 13641 & 0.88 & 7 & 16057 & 0.44 & $2.02(1.64,2.49)^{* * *}$ & $1.95(1.65,2.30)^{* * *}$ \\
\hline Yes & 64 & 23962 & 2.67 & 52 & 20165 & 2.58 & $1.04(0.90,1.20)$ & $1.10(0.98,1.25)$ \\
\hline \multicolumn{9}{|c|}{$\begin{array}{l}\text { Prostate cancer } \\
\text { screen }\end{array}$} \\
\hline \multicolumn{9}{|c|}{ Prostate biopsy } \\
\hline No & 47 & 36843 & 1.28 & 31 & 35516 & 0.87 & $1.46(1.29,1.66)^{* * *}$ & $1.43(1.28,1.60)^{* * *}$ \\
\hline Yes & 29 & 760 & 38.1 & 28 & 706 & 39.7 & $0.96(0.57,1.63)$ & $0.88(0.52,1.49)$ \\
\hline \multicolumn{9}{|l|}{ TURP } \\
\hline No & 61 & 36388 & 1.68 & 36 & 35109 & 1.03 & $1.64(1.44,1.85)^{* * *}$ & $1.53(1.38,1.70)^{* * *}$ \\
\hline Yes & 15 & 1216 & 12.3 & 23 & 1112 & 20.7 & $0.60(0.35,1.02)$ & $0.59(0.34,1.01)$ \\
\hline
\end{tabular}

Rate $^{\#}$, incidence rate per 1000 person-years; Crude HR, relative hazard ratio

Adjusted $\mathrm{HR}^{\dagger}$ : Multivariate analysis with adjustment for age; frequency of medical visits/per year; occupation; urbanization level; comorbidities of hyperlipidemia, diabetes, hypertension, urinary stones, urinary tract infection, obesity, anxiety, and depression; and prostate cancer screening strategies of prostate biopsy and TURP

Comorbidity*: Only one comorbidity (namely hyperlipidemia, diabetes, hypertension, urinary stones, urinary tract infection, coronary artery disease, and depression) classified as the comorbidity group. $P<.05,{ }^{* *} P<.01,{ }^{* * *} P<.001$

severity of $\mathrm{ED}$ and $\mathrm{PCa}$, in contrast to previous studies that used questionnaires to evaluate ED severity [23, 30, 31].

Second, the diagnoses of $\mathrm{ED}, \mathrm{PCa}$, and other comorbidities were based on ICD-9-CM codes; thus, misclassification is possible. However, the use of ICDCM-9 codes for diagnosing chronic diseases has been validated in previous nationwide cohort studies [32-36]. Moreover, the National Health Insurance Administration reviews charts, verifies medical charges, and imposes heavy penalties for inappropriate charges and malpractice. These checks and balances are assumed to ensure accurate coding [34, 37]. 
Table 3: Comparison of the incidence and hazard ratios of prostate cancer stratified by subtypes of erectile dysfunction

\begin{tabular}{lccccc}
\hline Variables & $\mathbf{N}$ & Event & Rate $^{\#}$ & $\begin{array}{c}\text { Crude HR } \\
\text { (95\% CI) }\end{array}$ & $\begin{array}{c}\text { Adjusted HR }^{\dagger} \\
\mathbf{( 9 5 \%} \text { CI) }\end{array}$ \\
\hline $\begin{array}{l}\text { Without erectile dysfunction } \\
\begin{array}{l}\text { Erectile dysfunction } \\
\text { Psychogenic ED }\end{array}\end{array}$ & 5857 & 59 & 1.63 & 1 (Reference) & 1 (Reference) \\
Organic ED & 579 & 3 & 0.76 & $0.44(0.14,1.43)$ & $0.43(0.13,1.37)$ \\
\hline
\end{tabular}

Rate $^{\#}$, incidence rate per 1000 person-years; Crude HR, relative hazard ratio

Adjusted $\mathrm{HR}^{\dagger}$ : Multivariate analysis adjusted for age; frequency of medical visits/per year; occupation; urbanization level; comorbidities of hyperlipidemia, diabetes, hypertension, urinary stones, urinary tract infection, obesity, anxiety, and depression; and prostate cancer screening strategies of prostate biopsy and TURP ***P<.001

Table 4: Trends of prostate cancer risks stratified by follow-up duration

\begin{tabular}{|c|c|c|c|c|c|c|c|c|}
\hline \multirow[b]{3}{*}{$\begin{array}{l}\text { Follow-up } \\
\text { time, years }\end{array}$} & \multicolumn{6}{|c|}{ Erectile dysfunction } & \multirow[b]{3}{*}{ Crude HR (95\% CI) } & \multirow[b]{3}{*}{$\begin{array}{c}\text { Adjusted HR }{ }^{\dagger} \\
(95 \% \text { CI) }\end{array}$} \\
\hline & & Yes & & & No & & & \\
\hline & Event & PY & Rate $^{\#}$ & Event & PY & Rate $^{\#}$ & & \\
\hline$\leq 1$ & 12 & 5824 & 2.06 & 5 & 5800 & 0.86 & $2.39(2.04,2.80)^{* * *}$ & $2.33(2.03,2.67)^{* * *}$ \\
\hline $2-3$ & 17 & 10383 & 1.64 & 11 & 10227 & 1.08 & $1.52(1.32,1.75)^{* * *}$ & $1.48(.30,1.67)^{* * *}$ \\
\hline $4-5$ & 17 & 8434 & 2.02 & 13 & 8159 & 1.59 & $1.27(1.09,1.47)^{* *}$ & $1.18(1.04,1.34)^{* *}$ \\
\hline$>5$ & 30 & 12963 & 2.31 & 30 & 12035 & 2.49 & $0.93(0.80,1.08)$ & $0.90(0.79,1.02)$ \\
\hline
\end{tabular}

Rate , incidence rate per 10,000 person-years; Crude HR, relative hazard ratio

Adjusted $\mathrm{HR}^{\dagger}$ : Multivariate analysis including age; frequency of medical visits/per year; occupation; urbanization level; comorbidities of hyperlipidemia, diabetes, hypertension, urinary stones, urinary tract infection, obesity, anxiety, and depression; and prostate cancer screening strategies of prostate biopsy and TURP $* * P<.01, * * * P<.001$

Moreover, to further reduce misclassification, the diagnosis of $\mathrm{PCa}$ was obtained from the Catastrophic Illness Patient Database, which includes proof of diagnosis by biopsy or tissue pathology. Because of the lack of crucial tumor characteristics in the database, such as stage and grade, we could not analyze the association between $\mathrm{ED}$ and $\mathrm{PCa}$ stage.

Third, the NHIRD lacks information on some critical ED risk factors such as smoking, obesity, BMI, alcoholism, exercise, and dietary habits. However, we have included hypertension, diabetes, and hyperlipidemia to adjust for the effect of BMI and obesity.

Our data suggest that patients with ED have an approximately 1.24-fold higher risk of subsequent $\mathrm{PCa}$ than do patients without ED, even for a follow-up duration of more than 5 years. For patients who initially present with ED, the evaluating physician should screen for $\mathrm{PCa}$, regardless of whether the patient is aged younger or older than 65 years and regardless of the presence or absence of any comorbidity. More research is needed - both regarding the possible biological link and to confirm the findings. In addition future studies should stratify the PCa according to risk class to assess any clinical significance.

\section{MATERIALS AND METHODS}

\section{Data source}

This retrospective cohort study used data from the Longitudinal Health Insurance Database 2000 (LHID2000). The LHID2000 is a subset of the National Health Insurance Research Database (NHIRD) and includes the health claims data of 1 million randomly selected beneficiaries of the Taiwan National Health Insurance (NHI) program for the period 1996-2011. To build the LHID2000, the National Health Research Institutes randomly selected 1 million beneficiaries from the NHIRD between 1996 and 2011. The details of the NHI program and LHID2000 have been described previously $[38,39]$. Disease classification in the LHID2000 is based on the International Classification of Diseases, Ninth Revision, Clinical Modification (ICD-9-CM). This study 
was approved by the Institutional Review Board of China Medical University (CMUH104-REC2-115-CR1).

\section{Sampled population}

To determine the relationship between ED and $\mathrm{PCa}$, we established an ED cohort and a non-ED cohort and observed the incidence of PCa. The ED cohort included men aged $\geqq 20$ years with new-onset ED including psychogenic ED (ICD-9-CM code 302.72) and organic ED (ICD-9-CM code 607.84) between January 1, 2000 and December 31,2010. The index date was the date of first ED diagnosis. To increase the diagnostic validity of ED, only those patients who had been diagnosed with ED at least twice in an outpatient service by urologists were included in the analysis.

The non-ED cohort included men without a history of ED who were randomly selected from the LHID2000. The non-ED cohort was frequency-matched to the ED cohort at a ratio of $1: 1$ by age (5-y intervals), frequency of medical visits/per year, occupation, urbanization level, index year, and comorbidities including hyperlipidemia, diabetes, hypertension, urinary stones, urinary tract infection, coronary artery disease, depression, prostate cancer screen, prostate biopsy, and TURP. The index date for the patients without ED was the same date as that for matched patients. This study excluded patients with a PCa diagnosis (ICD-9-CM code 185) before the index date. Both cohorts were followed until withdrawal from the NHI program, $\mathrm{PCa}$ event occurrence, or December 31, 2011.

\section{Variables of interest}

The sociodemographic variables used in this study were age, occupation category, and urbanization level. The details of occupation category and urbanization level are fully described in a previous study $[40,41]$. Pre-existing comorbidities included hyperlipidemia (ICD-9-CM code 272), diabetes (ICD-9-CM code 250), hypertension (ICD-9-CM codes 401-405), urinary stones (ICD-9-CM codes 592.0, 592.1, 594.0, and 594.1), urinary tract infection (ICD-9-CM codes 590 and 595), coronary artery disease (ICD-9-CM codes 410-414), and depression (ICD-9-CM codes 296.2-296.3, 300.4, and 311). Diagnosis of $\mathrm{PCa}$ according to tissues obtained from transurethral resection of the prostate (TURP) (ICD-9-procedure code 60.29) and prostate biopsy (ICD9-procedure codes 60.11 and 60.12).

\section{Statistical analysis}

The demographic and clinical characteristics of the ED and non-ED cohorts, including age, occupation category, urbanization level, comorbidities, and $\mathrm{PCa}$ screening strategies, were compared using the Chi-square test. Continuous variables were compared between the ED and non-ED cohorts by using the Student $t$ test. Cumulative incidence curves for PCa were plotted using the Kaplan-Meier method, and the curve differences between the cohorts were determined using the log-rank test. We calculated the incidence density rate of $\mathrm{PCa}$ (per 1000 person-y) for each cohort. The risk of $\mathrm{PCa}$ in the ED cohort compared with that in the non-ED cohort was presented as hazard ratios (HRs) and 95\% confidence intervals (CIs) by using univariate and multivariate Cox proportional hazards models. The multivariate Cox models were simultaneously adjusted for age; occupation category; urbanization level; comorbidities of hyperlipidemia, diabetes, hypertension, urinary stones, urinary tract infection, obesity, anxiety, and depression; and $\mathrm{PCa}$ screening strategies of prostate biopsy and TURP. All analyses were conducted using SAS statistical software (Version 9.4 for Windows; SAS Institute, Inc., Cary, NC, USA), with statistical significance set at $P<.05$ for a 2-tailed test.

\section{Author contributions}

The authors' individual contributions are outlined as follows.

Substantial contributions to conception and design: Wei-Yu Lin, Ying-Hsu Chang, Cheng-Li Lin, Chia-Hung Kao and His-Chin Wu,

Drafting and revising the article critically for important intellectual content: Wei-Yu Lin, Ying-Hsu Chang, Cheng-Li Lin, Chia-Hung Kao and His-Chin Wu,

Final approval of the version to be published: Wei-Yu Lin and His-Chin Wu.

\section{CONFLICT OF INTEREST}

All authors report no conflicts of interest.

\section{FUNDING}

This study is supported in part by Taiwan Ministry of Health and Welfare Clinical Trial and Research Center of Excellence (MOHW106-TDU-B-212-113004); China Medical University Hospital, Academia Sinica Taiwan Biobank, Stroke Biosignature Project (BM10501010037); NRPB Stroke Clinical Trial Consortium (MOST 1052325-B-039-003); Tseng-Lien Lin Foundation, Taichung, Taiwan; Taiwan Brain Disease Foundation, Taipei, Taiwan; Katsuzo and Kiyo Aoshima Memorial Funds, Japan; and Health, and welfare surcharge of tobacco products, China Medical University Hospital Cancer Research Center of Excellence (MOHW105-TDU-B-212-134003, Taiwan). The funders had no role in study design, data collection and analysis, decision to publish, or preparation of the manuscript. No additional external funding received for this study. 


\section{REFERENCES}

1. Siegel R, Naishadham D, Jemal A. Cancer statistics, 2012. CA Cancer J Clin. 2012; 62:10-29.

2. Sutcliffe S, Colditz GA. Prostate cancer: is it time to expand the research focus to early-life exposures? Nat Rev Cancer. 2013;13:208-518.

3. Cullen J, Elsamanoudi S, Brassell SA, Chen Y, Colombo M, Srivastava A, McLeod DG. The burden of prostate cancer in Asian nations. J Carcinog. 2012;11:7.

4. Pu YS, Chiang HS, Lin CC, Huang CY, Huang KH, Chen J. Changing trends of prostate cancer in Asia. Aging Male. 2004;7:120-132.

5. Chia SE, Tan CS, Lim GH, Sim X, Lau W, Chia KS. Incidence, mortality and five-year relative survival ratio of prostate cancer among Chinese residents in Singapore from 1968 to 2002 by metastatic staging. Ann Acad Med Singapore. 2010;39:466-471.

6. Williams S, Chiong E, Lojanapiwat B, Umbas R, Akaza H, Asian Oncology Summit 2013. Management of prostate cancer in Asia: resource-stratified guidelines from the Asian Oncology Summit 2013. Lancet Oncol. 2013; 14:e524-534.

7. Cuzick J, Thorat MA, Andriole G, Brawley OW, Brown PH, Culig Z, Eeles RA, Ford LG, Hamdy FC, Holmberg L, Ilic D, Key TJ, La Vecchia C, et al. Prevention and early detection of prostate cancer. Lancet Oncol. 2014; 15:e484-492.

8. Patel AR, Klein EA. Risk factors for prostate cancer. Nat Clin Pract Urol. 2009;6:87-95.

9. Leitzmann MF, Platz EA, Stampfer MJ, Willett WC, Giovannucci E. Ejaculation frequency and subsequent risk of prostate cancer. JAMA. 2004;291:1578-1586.

10. Dimitropoulou P, Lophatananon A, Easton D, Pocock R, Dearnaley DP, Guy M, Edwards S, O'Brien L, Hall A, Wilkinson R, Eeles R, Muir KR; UK Genetic Prostate Cancer Study Collaborators; British Association of Urological Surgeons Section of Oncology. Sexual activity and prostate cancer risk in men diagnosed at a younger age. BJU Int. 2009;103:178-185.

11. Chavez AH, Scott Coffield K, Hasan Rajab M, Jo C. Incidence rate of prostate cancer in men treated for erectile dysfunction with phosphodiesterase type 5 inhibitors: retrospective analysis. Asian J Androl. 2013;15:246-248.

12. Johannes $\mathrm{CB}$, Araujo AB, Feldman HA, Derby CA, Kleinman KP, McKinlay JB. Incidence of erectile dysfunction in men 40 to 69 years old: longitudinal results from the Massachusetts male aging study. J Urol. 2000;163:460-463.

13. Moinpour CM, Lovato LC, Thompson IM Jr, Ware JE Jr, Ganz PA, Patrick DL, Shumaker SA, Donaldson GW, Ryan A, Coltman CA Jr. Profile of men randomized to the prostate cancer prevention trial: baseline health-related quality of life, urinary and sexual functioning, and health behaviors. J Clin Oncol. 2000;18:1942-1953.
14. Thompson IM, Tangen CM, Goodman PJ, Probstfield JL, Moinpour CM, Coltman CA. Erectile dysfunction and subsequent cardiovascular disease. JAMA. 2005;294:2996-3002.

15. Ong WL, McLachlan H, Millar JL. Prevalence of Baseline Erectile Dysfunction (ED) in an Australian Cohort of Men with Localized Prostate Cancer. J Sex Med. 2015;12:1267-1274.

16. Kimura M, Banez LL, Gerber L, Qi J, Tsivian M, Freedland SJ, Satoh T, Polascik TJ, Baba S, Moul JW. Association between preoperative erectile dysfunction and prostate cancer features--an analysis from the Duke Prostate Center Database. J Sex Med. 2012; 9:1174-1181.

17. Chung SD, Kang JH, Liao CH, Chiu KM, Lin HC. Increased risk for cancer following erectile dysfunction: a nationwide population-based follow-up study. J Sex Med. 2011;8:1513-1520.

18. Society AC. Global Cancer Facts \& Figures. 2nd Edition. Atlanta, GA: American Cancer Society; 2011.

19. Katz DL. Lifestyle and dietary modification for prevention of heart failure. Med Clin North Am. 2004;88:1295-1320, xii.

20. Salomon G, Isbarn H, Budaeus L, Schlomm T, Briganti A, Steuber T, Heinzer H, Haese A, Graefen M, Karakiewicz PI, Huland H, Chun F. Importance of baseline potency rate assessment of men diagnosed with clinically localized prostate cancer prior to radical prostatectomy. J Sex Med. 2009;6:498-504.

21. Tamim HM, Mahmud S, Hanley JA, Boivin JF, Stang MR, Collet JP. Antidepressants and risk of prostate cancer: a nested case-control study. Prostate Cancer Prostatic Dis. 2008;11:53-60.

22. Bostwick DG, Burke HB, Djakiew D, Euling S, Ho SM, Landolph J, Morrison H, Sonawane B, Shifflett T, Waters DJ, Timms B. Human prostate cancer risk factors. Cancer. 2004;101:2371-2490.

23. Chung SD, Keller JJ, Lin HC. A case-control study on the association between chronic prostatitis/chronic pelvic pain syndrome and erectile dysfunction. BJU Int. 2012;110:726-730.

24. Dennis LK, Lynch CF, Torner JC. Epidemiologic association between prostatitis and prostate cancer. Urology. 2002;60:78-83.

25. Dennis LK, Dawson DV. Meta-analysis of measures of sexual activity and prostate cancer. Epidemiology. 2002;13:72-79.

26. De Marzo AM, Platz EA, Sutcliffe S, Xu J, Grönberg $\mathrm{H}$, Drake CG, Nakai Y, Isaacs WB, Nelson WG. Inflammation in prostate carcinogenesis. Nat Rev Cancer. 2007;7:256-269.

27. Pearce CL, Van Den Berg DJ, Makridakis N, Reichardt JK, Ross RK, Pike MC, Kolonel LN, Henderson BE. No association between the SRD5A2 gene A49T missense variant and prostate cancer risk: lessons learned. Hum Mol Genet. 2008;17:2456-2461. 
28. Scariano JK, Treat E, Alba F, Nelson H, Ness SA, Smith AY. The SRD5A2 V89L polymorphism is associated with severity of disease in men with early onset prostate cancer. Prostate. 2008;68:1798-1805.

29. Setiawan VW, Schumacher FR, Haiman CA, Stram DO, Albanes D, Altshuler D, Berglund G, Buring J, Calle EE, Clavel-Chapelon F, Cox DG, Gaziano JM, Hankinson SE, et al. CYP17 genetic variation and risk of breast and prostate cancer from the National Cancer Institute Breast and Prostate Cancer Cohort Consortium (BPC3). Cancer Epidemiol Biomarkers Prev. 2007;16:2237-2246.

30. Hao ZY, Li HJ, Wang ZP, Xing JP, Hu WL, Zhang TF, Zhang XS, Zhou J, Tai S, Liang CZ. The prevalence of erectile dysfunction and its relation to chronic prostatitis in Chinese men. J Androl. 2011;32:496-501.

31. Marszalek M, Wehrberger C, Hochreiter W, Temml C, Madersbacher S. Symptoms suggestive of chronic pelvic pain syndrome in an urban population: prevalence and associations with lower urinary tract symptoms and erectile function. J Urol. 2007; 177:1815-1819.

32. Su TW, Chou TY, Jou HJ, Yang PY, Lin CL, Sung FC, Hsu $\mathrm{CY}$, Kao CH. Peripheral Arterial Disease and Spinal Cord Injury: A Retrospective Nationwide Cohort Study. Medicine (Baltimore). 2015; 94:e1655.

33. Lin HJ, Weng SF, Yang CM, Wu MP. Risk of hospitalization for acute cardiovascular events among subjects with lower urinary tract symptoms: a nationwide population-based study. PLoS One. 2016;8:e66661.

34. Chen YC, Lin HY, Li CY, Lee MS, Su YC. A nationwide cohort study suggests that hepatitis $\mathrm{C}$ virus infection is associated with increased risk of chronic kidney disease. Kidney Int. 2014;85:1200-1207.
35. Chou TY, Su TW, Jou HJ, Yang PY, Chen HJ, Muo CH, Kao CH. Increased risk of peripheral arterial disease after hip replacement: an 11-year retrospective population-based cohort study. Medicine (Baltimore). 2015;94:e870.

36. Lin TY, Chen YG, Lin CL, Huang WS, Kao CH. Inflammatory Bowel Disease Increases the Risk of Peripheral Arterial Disease: A Nationwide Cohort Study. Medicine (Baltimore). 2015;94:e2381.

37. Sheu JJ, Kang JH, Lin HC, Lin HC. Hyperthyroidism and risk of ischemic stroke in young adults: a 5-year follow-up study. Stroke. 2010;41:961-966.

38. Kao CC, Lin CL, Huang WY, Cha TL, Lin TY, Shen $\mathrm{CH}$, Kao $\mathrm{CH}$. Association between inflammatory bowel disease and eredtile dysfunction: a nationwide populationbasedstudy. Inflamm Bowel Dis. 2016;22:1065-1070.

39. Chen $\mathrm{CH}$, Lin $\mathrm{CL}$, Kao $\mathrm{CH}$. Gastroesophageal reflux disease with proton pump inhibitor use is associated with an increased risk of osteoporosis: a nationwide populationbased analysis. Osteoporos Int. 2016;27:2117-2126.

40. Tu YF, Lin CL, Lin CH, Huang CC, Sung FC, Kao CH. Febrile convulsions increase risk of Tourette syndrome. Seizure. 2014; 23:651-656.

41. Chang YT, Chen PC, Tsai IJ, Sung FC, Chin ZN, Kuo $\mathrm{HT}$, Tsai $\mathrm{CH}$, Chou IC. Bidirectional relation between schizophrenia and epilepsy: a population-based retrospectivecohort study. Epilepsia. 2011 ;52:2036-2042. 